\title{
Cálculo da Solução Explícita de Controladores MPC por Modelo Takagi-Sugeno Modificado
}

\author{
Teófilo Paiva Guimarães Mendes*. Márcio André Fernandes Martins**. \\ Leizer Schnitman**.
}

\author{
*Universidade Federal do Recôncavo Baiano, Cruz das Almas, CEP 44380-000, Bahia, Brasil \\ (e-mail:teopgmendes@ufrb.edu.br). \\ **CTAI, Universidade Federal da Bahia, Salvador CEP 40210-630, Bahia, Brasil \\ (e-mail:marciomartins@ufba.br,leizer@ufba.br).
}

\begin{abstract}
Multi-parameter quadratic programming is a technique applied to compute optimization solution inherent to Model Predictive Control (MPC) strategies. The present work proposes a modified Takagi-Sugeno model to compute explicit solution, formed by the polyhedral critical regions and their respective affine functions. Advantages of this technique are: (i) capacity to complexity reduction e parallel processing, (ii) dismiss of model's training phase from numerical data; and (iii) synthesis of a single analytical expression for the associated MPC control law. A case study shows that this new method has potential to be more efficient than classical explicit MPC optimization techniques with respect to numbers of parameters and processing time.

Resumo: Programação multiparamétrica quadrática é uma técnica aplicada para calcular a solução da otimização inerente as estratégias de controle preditivo baseado em modelo (Model Predictive Control MPC). O presente trabalho propõe um modelo de Takagi-Sugeno modificado (TSM) para calcular a solução explícita MPC, formada pelas regiões críticas e suas respectivas funções afins. As vantagens desta técnica são: (i) capacidade de redução de complexidade e processamento paralelo, (ii) eliminar a fase de treinamento do modelo a partir de dados numéricos; e (iii) síntese de uma única expressão analítica para a lei de controle MPC associada. Um estudo de caso mostra que este novo método tem potencial para ser mais eficiente do que as técnicas clássicas de otimização explícitas de MPC com relação ao número de parâmetros e tempo de processamento.
\end{abstract}

Keywords: Control theory, model predictive control, multi-parameter programming, Takagi-Sugeno model, optimization in control and automation systems.

Palavras-chaves: Teoria de controle, controle preditivo baseado em modelo, programação multiparamétrica, modelo Takagi-Sugeno, otimização em sistemas de controle e automação.

\section{INTRODUÇÃO}

O termo MPC abrange a classe de estratégias de controle que usa modelos para prever a resposta de um sistema. A cada intervalo de amostragem, estas estratégias buscam otimizar o desempenho futuro do sistema, pelo cálculo de uma sequência futura de ações de controle. A primeira ação da sequência ótima é aplicada ao sistema, e a otimização é repetida no próximo instante (Qin e Badgwell, 2003).

Estratégias MPC com modelos e restrições lineares e com índices de desempenho quadráticos implicam em ter o termo linear deste índice e as restrições linearmente variantes com os estados do sistema. Neste caso, a programação quadrática usada para resolver a otimização pode ser substituída por uma programação quadrática multiparamétrica (multi-parameter quadratic programming - mpQP em inglês) (Bemporad et al. 2002). O algoritmo da mpQP consiste em, a partir de transformações lineares e condições de Karush-Kuhn-Tucker (KKT): dividir o espaço dos estados em poliedros formados pelas restrições ativas da otimização, ou regiões críticas; e encontrar funções afins nos estados que calculam a solução ótima localmente em cada região crítica. Logo, este método computacional cria uma tabela de consulta em que SE o estado pertence a uma região, ENTÃO a ação correspondente a essa região é executada. Em razão desta lei de controle ser conhecida a priori, ou off-line, a solução ótima dada pela mpQP é chamada de explícita. Num dado instante, os cálculos on-line destas estratégias são reduzidos a: localizar os estados, que equivalem a um ponto, em uma das regiões críticas; e aplicar a respectiva função afim local.

Estratégias MPC com solução explícita são próprias para aplicações com dinâmicas rápidas, na ordem de 1 a $50 \mathrm{~ms}$, e em sistemas relativamente pequenos, com 1 a 2 entradas de controle e de 5 a 10 parâmetros. Contudo, existe clara limitação deste método. O aumento da dimensão do problema de otimização, especialmente do número de restrições, leva a um crescimento expressivo do número de regiões que compõem a solução explícita (Alessio e Bemporad, 2009). 
Recentemente, o artigo de Oberdieck et al. (2016) revisou e discutiu os principais desenvolvimentos na área de algoritmos mpQP ao longo das últimas duas décadas. Esse trabalho levantou pesquisas que procuraram contornar as limitações do método de otimização multiparamétrica, classificando-as em duas linhas. A primeira estuda meios de reduzir a complexidade das regiões críticas, para diminuir quantidade de parâmetros. A segunda busca o desenvolvimento de algoritmos mais eficientes de localização de pontos interiores, para diminuir o número de operações matemáticas. Nenhuma das pesquisas levantadas usou métodos de inteligência artificial (IA) com estes objetivos, exceto o trabalho de Cseko et al. (2015). Este autor aproximou solução explícita por uma rede neural artificial (RNA), em troca da redução da complexidade das regiões críticas.

No presente artigo, a solução explícita de estratégias MPC é calculada por outra abordagem. Propõe-se um modelo Takagi-Sugeno (1993) contendo uma nova camada de entrada para lidar com as partições poliédricas formadas pelas regiões críticas. Portanto, ele é chamado de modelo Takagi-Sugeno modificado (TSM). O novo método considera os parâmetros da mpQP como variáveis de entrada, as regiões críticas como os conjuntos dos termos antecedentes, a função limiar nas funções de pertinência e as funções afins locais nos termos do consequente do modelo TSM.

O sistema de inferência do modelo TSM propicia um modo direto de reduzir a complexidade das regiões críticas. Isso é alcançado usando conectivos lógicos nas regras SE-ENTÃO com o mesmo consequente. Além disso, ele é eficiente para localizar o estado em uma das regiões críticas. Isso se deve ao cálculo paralelo realizado no antecedente. O método proposto não se trata de uma aproximação, e sim de uma nova estrutura para calcular a solução explícita MPC. Logo, tem como vantagem não necessitar de treinamento a partir de dados para encontrar parâmetros do modelo. Outro vantagem do método proposto é possibilitar a síntese de uma única expressão analítica da função afim por partes.

O restante deste artigo é organizado do seguinte modo. A fundamentação teórica sobre estratégias MPC é revisada na seção 2 e sobre modelos TS na seção 3. O modelo TSM proposto é detalhado na seção 4. Um estudo de caso é apresentado e seus resultados discutidos na seção 5. Por fim, a conclusão e trabalhos futuros são apresentados na seção 6 .

\section{MPC COM SOLUÇÃO EXPLÍCITA}

\subsection{Formulação do problema de otimização MPC}

Seja um sistema dinâmico modelado pela equação de estados em (1) e com restrições dadas por (2):

$$
\begin{gathered}
\boldsymbol{x}_{k+1}=\boldsymbol{A} \boldsymbol{x}_{k}+\boldsymbol{B} \boldsymbol{u}_{k} \\
\boldsymbol{y}_{k}=\boldsymbol{C} \boldsymbol{x}_{k} \\
\boldsymbol{u}_{\text {min }} \leq \boldsymbol{u}_{k+n} \leq \boldsymbol{u}_{\text {max }} \\
\boldsymbol{y}_{\text {min }} \leq \boldsymbol{y}_{k+n} \leq \boldsymbol{y}_{\text {max }}
\end{gathered}
$$

na qual $\boldsymbol{x}_{k}, \boldsymbol{u}_{k}$ e $\boldsymbol{y}_{k}$ são os vetores de estado, entradas e saídas, respectivamente de dimensões $n_{x}, n_{u}$ e $n_{y}, \boldsymbol{A}$ é estável e o par $(A, B)$ é controlável.

Considere o caso regulador de controle, e o índice de desempenho quadrático $J$ em (3).

$$
J=\left\|\boldsymbol{x}_{k+N / t}\right\|_{\boldsymbol{P}}^{2}+\sum_{n=1}^{N}\left\|\boldsymbol{x}_{k+n / t}\right\|_{\boldsymbol{Q}}^{2}+\sum_{n=0}^{N-1}\left\|\boldsymbol{u}_{k+n / t}\right\|_{\boldsymbol{R}}^{2}
$$

em que a notação \|\| representa a norma euclidiana, as matrizes $\boldsymbol{P}, \boldsymbol{Q}$ e $\boldsymbol{R}$ representam ponderações dos estados e saídas nesta norma, e $N$ o horizonte de controle.

Note que em (3) foi considerado horizonte infinito de predição dos estados para garantir a estabilidade do sistema em malha fechada. Portanto, $\boldsymbol{P}$ é solução da equação de Lyapunov $\boldsymbol{P}=\boldsymbol{A}^{T} \boldsymbol{P A}+\boldsymbol{Q}$.

A estratégia MPC consiste em calcular a resposta do problema em (4) a cada intervalo de controle.

$$
\begin{aligned}
& \min _{\boldsymbol{u}_{k}, . . \boldsymbol{u}_{k+N-1}} J\left(\boldsymbol{u}_{k}, \ldots \boldsymbol{u}_{k+N-1}, \boldsymbol{x}_{k}\right) \\
& \text { sujeito } a:
\end{aligned}
$$$$
\text { (1) }-(2)
$$

Reescrevendo (4) em notação matricial, padrão utilizado em algoritmos de otimização, tem-se (5):

$$
\begin{aligned}
& \min _{\boldsymbol{u}} J=\boldsymbol{u}^{\mathbf{T}} \boldsymbol{H} \boldsymbol{u}+2 \boldsymbol{x}_{k}^{T} \boldsymbol{F} \boldsymbol{u}+\boldsymbol{x}_{k}^{T} \boldsymbol{Y} \boldsymbol{x}_{k} \\
& \text { sujeito } a: \\
& \boldsymbol{G u} \leq \boldsymbol{W}+\boldsymbol{E} \boldsymbol{x}_{k}
\end{aligned}
$$

em que $\boldsymbol{u}=\left[\begin{array}{lll}\boldsymbol{u}_{k} & \ldots & \boldsymbol{u}_{k+N-1}\end{array}\right]^{\boldsymbol{T}}$, e as matrizes $\boldsymbol{H}, \boldsymbol{F}, \boldsymbol{Y}, \boldsymbol{G}, \boldsymbol{W}$, e $\boldsymbol{E}$ são encontradas a partir de (1), (2) e (3).

\subsection{Solução explícita e algoritmo $m p Q P$}

O conceito matemático que embasa a solução explícita é mostrado a seguir. Recomenda-se a leitura de Bemporad et al. (2002) para maiores detalhes.

Os algoritmos mpQP partem da substituição da transformação linear em (6) no problema em (5).

$$
\boldsymbol{z}=\boldsymbol{u}+\boldsymbol{H}^{-1} \boldsymbol{F} \boldsymbol{x}_{k}
$$

Deste modo, o problema a ser resolvido passa a ser dado por (7), em que $\boldsymbol{S}=\boldsymbol{E}+\boldsymbol{G} \boldsymbol{H}^{-1} \boldsymbol{F} \boldsymbol{T}$.

$$
\begin{aligned}
& \min _{\mathbf{z}} J=\boldsymbol{z}^{\mathbf{T}} \boldsymbol{H} \boldsymbol{z} \\
& \text { sujeito } a: \\
& \boldsymbol{G} \boldsymbol{z} \leq \boldsymbol{W}+\boldsymbol{S} \boldsymbol{x}_{k}
\end{aligned}
$$

Note que o índice de desempenho $J$ passa a ser função apenas de $\boldsymbol{z}$, e os estados $\boldsymbol{x}_{k}$ aparecem apenas do lado direito das restrições. Assim, assumindo $\mathrm{H}$ como sendo definida positiva, as condições de otimalidade KKT de primeira ordem de (7) são dadas por (8):

$$
\boldsymbol{H} \boldsymbol{z}+\boldsymbol{G}^{T} \boldsymbol{\lambda}=0
$$




$$
\begin{gathered}
\boldsymbol{G} \boldsymbol{z} \leq \boldsymbol{W}+\boldsymbol{S} \boldsymbol{x}_{k}, \\
\lambda_{l}\left(\boldsymbol{G}^{l} \boldsymbol{z}-\boldsymbol{W}^{l}-\boldsymbol{S}^{l} \boldsymbol{x}_{k}\right)=0, \\
\lambda_{l} \geq 0, \quad l=1, \ldots q,
\end{gathered}
$$

na qual $\lambda \in \mathfrak{R}^{q}$ é o vetor das $\lambda_{l}$ multiplicadores KKT, e $l$ indica a respectiva linha das matrizes $\boldsymbol{G}^{l}, \boldsymbol{W}^{l}$, e $\boldsymbol{E}^{l}$.

Seja $z^{*}\left(\boldsymbol{x}_{k}\right)$ a solução ótima do problema (7). A partir de (8a), é possível encontrar (9).

$$
z^{*}\left(\boldsymbol{x}_{k}\right)=-\boldsymbol{H}^{-1} \boldsymbol{G}^{T} \lambda
$$

Sejam também $\tilde{\lambda}>0$ os multiplicadores KKT e " " o índice referentes as linhas não nulas em (8c). Assim, têm-se as respectivas restrições ativas na forma em (10).

$$
\tilde{\boldsymbol{G}}^{*}\left(\boldsymbol{x}_{k}\right)-\tilde{\boldsymbol{W}}-\tilde{\boldsymbol{S}} \boldsymbol{x}_{k}=0
$$

Assume-se as linhas de $\tilde{\boldsymbol{G}}$ linearmente independentes. Assim, substituindo as linhas não nulas de (9) em (10), tem-se $\tilde{\lambda}$ apenas em função de $\boldsymbol{x}_{k}$ em (11).

$$
\tilde{\lambda}=-\left(\tilde{\boldsymbol{G}} \boldsymbol{H}^{-1} \tilde{\boldsymbol{G}}^{T}\right)^{-1}\left(\tilde{\boldsymbol{W}}+\tilde{\boldsymbol{S}} \boldsymbol{x}_{k}\right)
$$

Substituindo (11) em (9), tem-se $z^{*}$ também apenas em função dos parâmetros $\boldsymbol{x}_{k} \mathrm{em}$ (12).

$$
z^{*}\left(\boldsymbol{x}_{k}\right)=\boldsymbol{H} \tilde{\boldsymbol{G}}^{T}\left(\tilde{\boldsymbol{G}} \boldsymbol{H} \tilde{\boldsymbol{G}}^{T}\right)^{-1}\left(\tilde{\boldsymbol{W}}+\tilde{\boldsymbol{S}} \boldsymbol{x}_{k}\right)
$$

A solução do problema de otimização MPC, em sua variável de decisão original $\mathbf{u}^{*}$, é encontrada substituindo (12) em (6), encontrando-se (13).

$$
\mathbf{u}^{*}=\boldsymbol{H} \tilde{\boldsymbol{G}}^{T}\left(\tilde{\boldsymbol{G}} \boldsymbol{H} \tilde{\boldsymbol{G}}^{T}\right)^{-1}\left(\tilde{\boldsymbol{W}}+\tilde{\boldsymbol{S}} \boldsymbol{x}_{k}\right)-\boldsymbol{H}^{-1} \boldsymbol{F} \boldsymbol{x}_{k}
$$

Note que (13) é válida apenas na região crítica $C R_{i}$, determinada pela substituição de (9) e (11) respectivamente em (8b) e (8d), encontrando (14).

$$
\left[\begin{array}{c}
\boldsymbol{G} \boldsymbol{H} \boldsymbol{G}^{T}\left(\tilde{\boldsymbol{G}} \boldsymbol{H}^{-1} \tilde{\boldsymbol{G}}^{T}\right)^{-1}\left(\tilde{\boldsymbol{W}}+\tilde{\boldsymbol{S}} \boldsymbol{x}_{k}\right) \\
\left(\tilde{\boldsymbol{G}} \boldsymbol{H} \tilde{\boldsymbol{G}}^{T}\right)^{-1}\left(\tilde{\boldsymbol{W}}+\tilde{\boldsymbol{S}} \boldsymbol{x}_{k}\right)
\end{array}\right] \leq\left[\begin{array}{c}
\boldsymbol{W}+\boldsymbol{S} \boldsymbol{x}_{k} \\
0
\end{array}\right]
$$

Após a eliminação de linhas redundantes dessas inequações, tem-se as regiões críticas definidas em (15), e as respectivas funções afim locais em (16):

$$
\begin{gathered}
\boldsymbol{M}^{i} \boldsymbol{x}_{k} \leq \boldsymbol{b}^{i} \\
\boldsymbol{u}_{i}^{*}=\boldsymbol{K}^{i} \boldsymbol{x}_{k}+\boldsymbol{h}^{i}
\end{gathered}
$$

em que $\boldsymbol{M}^{i}, \boldsymbol{b}^{i}, \boldsymbol{K}^{i}$ e $\boldsymbol{k}^{i}$ são encontradas a partir de (13) e (14), além de $\boldsymbol{M}^{i}, \boldsymbol{b}^{i}$ possuírem $r_{\text {imax }}$ linhas correspondentes a quantidade de desigualdades que definem a respectiva $C R_{i}$.

Um algoritmo de programação multiparamétrica é descrito em Bemporad et al. (2002). Ele obtém sistematicamente todas as $p$ regiões críticas que compõem o espaço de soluções viáveis de (5). Após aplicação deste, ou outro algoritmo de $\mathrm{mpQP}$, encontra-se a solução explícita em (17).

$$
\left\{\begin{array}{cccc}
\text { Se } & \boldsymbol{x}_{k} \in C R_{l} & \text { então } & \boldsymbol{u}^{*}\left(\boldsymbol{x}_{k}\right)=\boldsymbol{K}^{l} \boldsymbol{x}_{k}+\boldsymbol{h}^{l} \\
\vdots & \vdots & \vdots & \vdots \\
\text { Se } & \boldsymbol{x}_{k} \in C R_{p} & \text { então } & \boldsymbol{u}^{*}\left(\boldsymbol{x}_{k}\right)=\boldsymbol{K}^{p} \boldsymbol{x}_{k}+\boldsymbol{h}^{p}
\end{array}\right.
$$

\section{MODELO TAKAGI-SUGENO}

Considere um modelo TS composto por $p$ regras SEENTÃO, escolhido para aproximar um sistema usando lógica aproximada (rough) ou nebulosa (fuzzy). A $i$-ésima regra $R_{i}$ deste modelo será da forma:

$$
R_{i}:\left\{\begin{array}{l}
S e \quad x_{1} \text { é } A_{i, 1} E x_{2} e ́ A_{l, 2} \\
\text { Então } \quad y=f_{i}\left(x_{1}, x_{2} \ldots x_{n}\right)
\end{array}\right.
$$

em que $x_{i} \in \Re$ são as variáveis de entrada, $A_{i, j}$ são os conjuntos aproximados, parte do antecedente, $f_{i}$ são as funções locais dos consequentes e $y \in \mathfrak{R}^{n u}$ a saída final do modelo.

No antecedente deste tipo de modelo, pode-se escolher partições de formato retangular em grelha, em árvore e em dispersão, ou de formatos elipsóides oblíquas. Independente do tipo de partição, o sistema de inferência do modelo TS calcula a saída em camadas, conforme explicado a seguir, e mostrado na Figura 1.

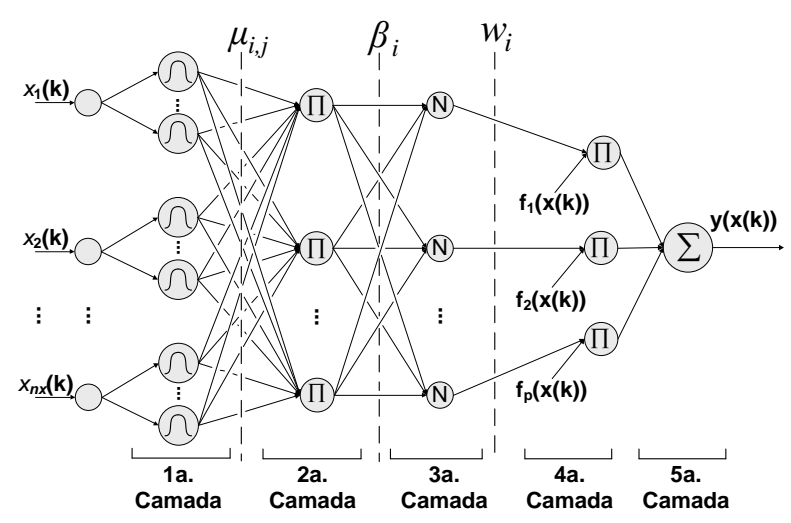

Figura 1- Sistema de inferência do modelo TS clássico.

Na primeira camada, é verificada a pertinência $\mu_{i, j}$ de cada variável $x_{j}(k)$, com $i=1, \ldots p$ e $j=1, \ldots n_{x}$. Pode-se usar, por exemplo, a função limiar como em (19).

$$
\mu_{i, j}\left(x_{j}\right)=0,5 .\left(1-\frac{x_{j}}{\left|x_{j}\right|}\right)=\left\{\begin{array}{ccc}
1 & \text { se } & x_{j}<0 \\
0,5 & \text { se } & x_{j}=0 \\
0 & \text { se } & x_{j}>0
\end{array}\right.
$$

Encontram-se os pesos das regras $\beta_{i}\left(\boldsymbol{x}_{k}\right)$ na segunda camada por (20), e os pesos normalizados $w_{i}\left(\boldsymbol{x}_{k}\right)$ na terceira camada por (21).

$$
\begin{gathered}
\beta_{i}\left(\boldsymbol{x}_{k}\right)=\prod_{j=1}^{n_{x}} \mu_{i, j}\left(x_{j}(k)\right) \\
w_{i}\left(\boldsymbol{x}_{k}\right)=\beta_{i}\left(\boldsymbol{x}_{k}\right) /\left(\sum_{i=1}^{p} \beta_{i}\left(\boldsymbol{x}_{k}\right)\right)
\end{gathered}
$$


Pondera-se cada função local $f_{i}\left(\boldsymbol{x}_{k}\right)$ pelo respectivo peso normalizado $w_{i}\left(\boldsymbol{x}_{k}\right)$ na quarta camada. Por fim, somam-se as contribuições individuais da camada anterior para obter a saída do modelo $y\left(\boldsymbol{x}_{k}\right)$ na quinta camada, conforme (22).

$$
y\left(\boldsymbol{x}_{k}\right)=\sum_{i=1}^{p} w_{i}\left(\boldsymbol{x}_{k}\right) f_{i}\left(\boldsymbol{x}_{k}\right)
$$

\section{MÉTODO PROPOSTO}

\subsection{Antecedente do modelo TSM}

O modelo TS tem capacidade de lidar com diferentes tipos de partições e exibe propriedades de aproximador universal. Contudo, a natureza das regiões críticas se coloca como desafio de modelagem para este método. Isso ocorre ainda que este use regras SE-ENTÃO como a solução explícita.

Este problema acontece devido as partições retangulares e elípticas não serem capazes de representar as partições poliédricas com exatidão. Métodos baseados nestes tipos de partições sempre exibem erros de aproximação, que podem ser reduzidos pelo aumento de regras.

Como a solução explícita é conhecida, é natural que seja modelada sem erros. Para tal, propõe-se o modelo TSM, o qual consiste do modelo TS clássico com uma nova camada de entrada. A função desta nova camada é calcular variáveis de particionamento $z_{i, j}\left(\boldsymbol{x}_{k}\right)$, conforme (23):

$$
z_{i j}\left(\boldsymbol{x}_{k}\right)=\boldsymbol{m}_{j}^{i} \boldsymbol{x}_{k}-b_{j}^{i} \leq 0
$$

em que os termos $\boldsymbol{m}_{j}^{i}$ e $b_{j}^{i}$ referem-se as linhas $j=1, \ldots r_{\text {imax }}$ das matrizes $\boldsymbol{M}^{i}, \boldsymbol{b}^{i}$ em (15), ressaltando que $r_{\text {imax }}$ varia entre $C R_{i} \mathrm{~s}$.

Sejam os conjuntos aproximados $A_{i, j}$ no antecedente do TSM definidos como em (24).

$$
A_{i, j}=\left\{z_{i, j}, \mu_{i, j}\left(z_{i, j}\right) \in \mathfrak{R} \mid z_{i, j} \leq 0, \mu_{i, j}\left(z_{i, j}\right) \in[0,1]\right\}
$$

Propõe-se representar as funções de pertinência $\mu_{i, j}\left(z_{i, j}\left(\boldsymbol{x}_{k}\right)\right)$ pela função limiar, como em (25).

$$
\mu_{i, j}\left(z_{i, j}\right)=0,5 .\left(1-\frac{z_{i, j}}{\left|z_{i, j}\right|}\right)=\left\{\begin{array}{ccc}
1 & \text { se } & z_{i, j}<0 \\
0,5 & \text { se } & z_{i, j}=0 \\
0 & \text { se } & z_{i, j}>0
\end{array}\right.
$$

Note que cada função de pertinência $\mu_{i, j}\left(z_{i, j}\left(\boldsymbol{x}_{k}\right)\right)$ em (25) depende de todos elementos do vetor $\boldsymbol{x}_{k}$. Porém, cada função $\mu_{i, j}\left(x_{j}(k)\right)$ em (20) depende apenas de um elemento $x_{j}(k)$.

\subsection{Formação das regras e consequente}

Uma região crítica $C R_{i}$ é determinada no modelo TSM pela interseção dos conjuntos $A_{i, j}$, como em (26).

$$
C R_{i}=\bigcap_{j=1}^{r_{i \max }} A_{i, j}
$$

A operação de intersecção entre conjuntos é equivalente ao operador lógico E. Escolhendo a função produto para aplicar este operador, tem-se o peso de cada regra $\beta_{i}\left(\mathbf{x}_{k}\right)$ em (27):

$$
\beta_{i}\left(\boldsymbol{x}_{k}\right)=\prod_{j=1}^{r_{i \max }} \mu_{i, j}\left(\boldsymbol{x}_{k}\right)=\left\{\begin{array}{ccc}
1 & \text { se } & \boldsymbol{x}_{k} \in C R_{i} \\
0 & \text { se } & \boldsymbol{x}_{k} \notin C R_{i} \\
0,5^{n} & \text { se } & \boldsymbol{x}_{k} \in C R_{i} e C R_{m}
\end{array}\right.
$$

em que $n$ é o número de $C R_{i} \mathrm{~s}$ as quais $\boldsymbol{x}_{k}$ pertence, e $m=1, \ldots n$. Os pesos normalizados $w_{i}\left(\mathbf{x}_{k}\right)$ são calculados por (28).

$$
w_{i}\left(\boldsymbol{x}_{k}\right)=\beta_{i}\left(\boldsymbol{x}_{k}\right) /\left(\sum_{i=1}^{p} \beta_{i}\left(\boldsymbol{x}_{k}\right)\right)
$$

Propõe-se usar a mesma função local da solução explícita em (16) no consequente do modelo TSM. Logo, tem-se as $R_{i}$ regras SE-ENTÃO deste modelo dadas por (29):

$$
R_{i}:\left\{\begin{array}{lc}
S e & z_{i, 1}\left(\boldsymbol{x}_{k}\right) \in A_{i, 1} E \ldots E z_{i, r_{i \max }}\left(\boldsymbol{x}_{k}\right) \in A_{r_{i \max }} \\
\text { Então } & \boldsymbol{x}_{k} \in C R_{i} \quad E \quad \boldsymbol{u}_{i}^{*}\left(\boldsymbol{x}_{k}\right)=\boldsymbol{K}^{i} \boldsymbol{x}_{k}+\boldsymbol{h}^{i}
\end{array}\right.
$$

A saída final do modelo TSM é calculada de modo equivalente a (22). Ponderam-se as funções locais pelos respectivos pesos normalizados das regras, como em (30).

$$
\boldsymbol{u}^{*}\left(\boldsymbol{x}_{k}\right)=\sum_{i=1}^{p} w_{i}\left(\boldsymbol{x}_{k}\right) \boldsymbol{u}_{i}^{*}\left(\boldsymbol{x}_{k}\right)
$$

O sistema de inferência do modelo TSM é representado graficamente na Figura 2, na qual é destacada a nova camada que calcula as variáveis de particionamento $z_{i, j}\left(\boldsymbol{x}_{k}\right)$.

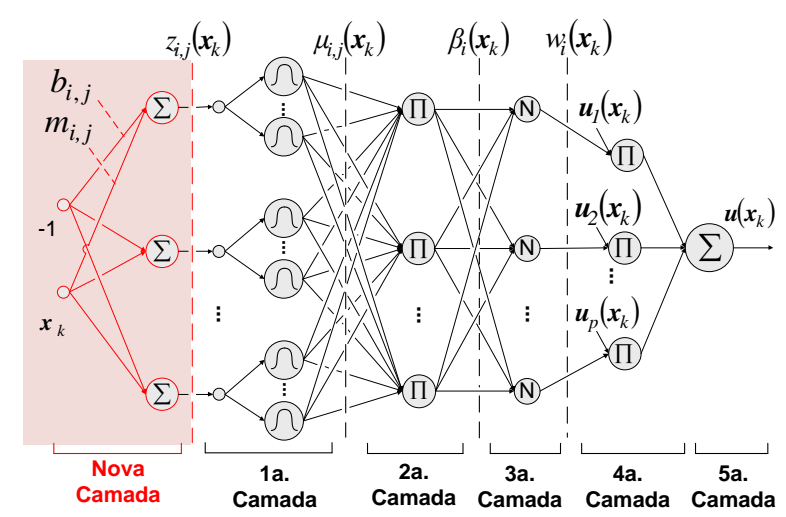

Figura 2- Sistema de inferência do modelo TSM.

\subsection{Observações sobre o modelo TSM}

A função em (25) assume três valores distintos, cada qual com uma interpretação. Quando a função pertinência $\mu_{i, j}\left(\boldsymbol{x}_{k}\right)$ tem valor 1 , o estado $\boldsymbol{x}_{k}$ pertence ao conjunto $A_{i, j}$. Quando a função pertinência $\mu_{i, j}\left(\boldsymbol{x}_{k}\right)$ tem valor 0 , o estado $\boldsymbol{x}_{k}$ não pertence ao conjunto $A_{i, j}$. Quando a função pertinência $\mu_{i, j}\left(\boldsymbol{x}_{k}\right)$ tem valor de 0,5 , o estado $\boldsymbol{x}_{k}$ está na fronteira de $A_{i, j}$. É importante notar que, nessa última situação, uma outra função de pertinência $l \neq j$, de outra região crítica $m \neq i$, também terá valor de 0,5 . Ou seja, este último caso sempre ocorre num par de conjuntos aproximados $A_{i, j}$ e $A_{l, m}$. 
Do mesmo modo, existem três situações distintas ao se calcular os pesos das regras $\beta_{i}\left(\boldsymbol{x}_{k}\right)$ por (27). Cada uma delas tem um significado associado ao pertencimento de $\boldsymbol{x}_{k}$ aos conjuntos $A_{i, j}$ que formam a região crítica $C R_{i}$ em (26). No primeiro caso, o estado $\boldsymbol{x}_{k}$ pertence a todos conjuntos $A_{i, j}$. Assim, as $r_{\text {imax }}$ funções de pertinência $\mu_{i, j}\left(\boldsymbol{x}_{k}\right)$ do antecedente da regra $i$ terão valor 1 . Portanto, o peso $\beta_{i}\left(\boldsymbol{x}_{k}\right)$ dessa regra também será 1 . No segundo caso, o estado $\boldsymbol{x}_{k}$ não pertence a apenas um dos conjuntos $A_{i, j}$. Logo, a função de pertinência deste conjunto terá valor 0 . Por consequência, o peso $\beta_{i}\left(\boldsymbol{x}_{k}\right)$ da respectiva regra $i$ também será 0 . No terceiro e último caso, o estado $\boldsymbol{x}_{k}$ pode pertence a fronteira compartilhada entre uma ou mais regiões críticas. Ou seja, este $\boldsymbol{x}_{k}$ pertence tanto a $C R_{i}$ quanto a $C R_{m}$. Assim, uma ou mais funções de pertinência $\mu_{i, j}\left(\boldsymbol{x}_{k}\right)$ e $\mu_{m, l}\left(\boldsymbol{x}_{k}\right)$ das respectivas regiões criticas terão valores de 0,5 . Deste modo, isso leva cada peso de regra $\beta_{i}\left(\boldsymbol{x}_{k}\right)$ e $\beta_{m}\left(\boldsymbol{x}_{k}\right)$ a ter valor de $0,5^{n}$. Ressalta-se que, neste último caso, as funções locais tem o mesmo valor nas fronteiras das regiões críticas. Isso ocorre em soluções explícitas calculadas por funções afim por partes contínuas. Considerando estas três situações, tem-se ainda que os pesos normalizados $w_{i}\left(\mathbf{x}_{k}\right)$ são iguais aos pesos das regras $\beta_{i}\left(\boldsymbol{x}_{k}\right)$.

Por fim, com base nos valores numéricos possíveis, e substituindo as equações (23), (25), (27) e (28) em (30), encontra-se uma única expressão analítica em (31).

$$
\boldsymbol{u}^{*}\left(\boldsymbol{x}_{k}\right)=\sum_{i=1}^{p}\left\{\left[\prod_{j=1}^{r_{i \max }} \frac{1}{2}\left(1-\frac{m_{i, j} \boldsymbol{x}_{k}-b_{i, j}}{\left|m_{i, j} \boldsymbol{x}_{k}-b_{i, j}\right|}\right)\right]\left[\boldsymbol{K}^{i} \boldsymbol{x}_{k}+\boldsymbol{h}^{i}\right]\right\}
$$

\section{ESTUDO DE CASO}

Seja o sistema dinâmico descrito pela equação de estado em (32), sujeito as restrições de entradas $\boldsymbol{u}_{k} \leq|2|$.

$$
\begin{gathered}
\boldsymbol{x}_{k+1}=\left[\begin{array}{cc}
0,7326 & -0,0861 \\
0,1722 & 0,9909
\end{array}\right] \boldsymbol{x}_{k}+\left[\begin{array}{c}
0,0609 \\
0,0064
\end{array}\right] \boldsymbol{u}_{k} \\
\boldsymbol{y}_{k}=\left[\begin{array}{ll}
0 & 1,4142
\end{array}\right] \boldsymbol{x}_{k}
\end{gathered}
$$

Considere a estratégia MPC formulada para o caso regulador em (5), e parâmetros de sintonia escolhidos em (33), assim como no estudo de caso em Bemporad et al. (2002).

$$
\boldsymbol{Q}=I_{2 \times 2}, \quad \boldsymbol{R}=0,01, \quad \quad N=2
$$

Aplicando um algoritmo mpQP, encontra-se a função afim por partes da Figura 3. As equações (34), (35), (36) e (37) são indicadas como exemplo para, respectivamente, encontrar as funções locais $\boldsymbol{u}_{4}, \boldsymbol{u}_{6}, \boldsymbol{u}_{7}$ e as regiões criticas $C R_{4}, C R_{6}$ e $C R_{7}$.

$$
\begin{gathered}
\boldsymbol{u}_{4}\left(\boldsymbol{x}_{k}\right)=\boldsymbol{u}_{6}\left(\boldsymbol{x}_{k}\right)=\boldsymbol{u}_{7}\left(\boldsymbol{x}_{k}\right)=\left[\begin{array}{ll}
0 & 0
\end{array}\right] \boldsymbol{x}_{k}+2 \\
C R_{4}:\left[\begin{array}{cc}
-0,5926 & 0,8055 \\
0,8072 & 0,5902
\end{array}\right] \boldsymbol{x}_{k} \leq\left[\begin{array}{c}
-0,2370 \\
-0,1708
\end{array}\right] \\
C R_{6}:\left[\begin{array}{cc}
-0,5926 & 0,8055 \\
0,6522 & 0,7581 \\
0,5926 & -0,8055
\end{array}\right] \boldsymbol{x}_{k} \leq\left[\begin{array}{c}
0,4571 \\
-0,2203 \\
0,2370
\end{array}\right]
\end{gathered}
$$

São necessários 99 parâmetros para representar a solução explícita da Figura 3. Este número é calculado pela soma dos 72 parâmetros das regiões críticas, na forma de (15), e dos 27 parâmetros das funções locais, na forma de (16). O modelo TSM necessita deste mesmo número de parâmetros.

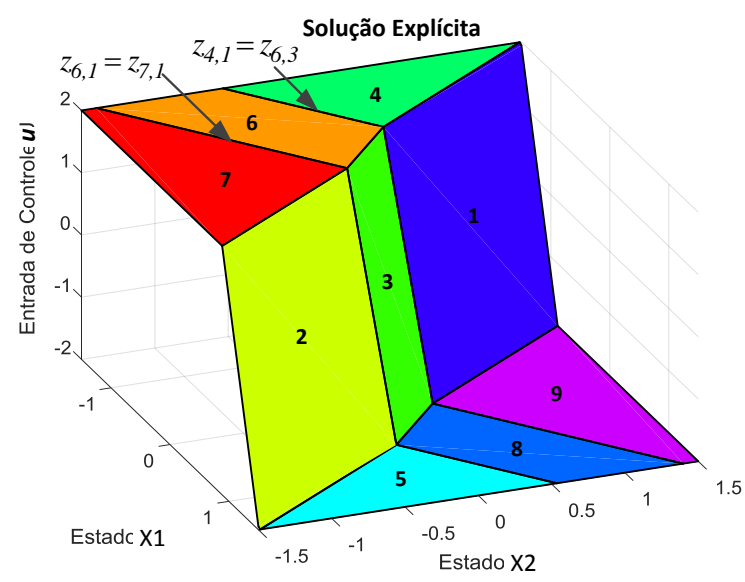

Figura 3- Solução explícita para $N=2$.

Note que, na Figura 3, existem 9 regiões críticas, as quais são identificadas por índices numéricos. Porém, existem apenas 5 funções locais distintas, havendo saturação da lei de controle em $\boldsymbol{u}_{4,6,7}=\boldsymbol{u}_{\text {max }}$ e $\boldsymbol{u}_{5,8,9}=\boldsymbol{u}_{\text {min }}$. Portanto, é importante reduzir a complexidade desta resposta e, por consequência, eliminar os parâmetros e operações matemáticas desnecessários.

A partir da solução explícita encontrada, a redução de complexidade é alcançada unindo regiões críticas com mesma função local e que possam formar poliedros convexos. Assim, escreve-se a união das regiões pela notação de inequações em (15), removendo as linhas repetidas. Neste estudo de caso, é possível unir as regiões críticas $C R_{6}$ com $C R_{7}$ e $C R_{8}$ com $C R_{9}$. O número de parâmetros após esta redução de complexidade é mostrado na Tabela 1 em [1].

A redução de complexidade no modelo TSM é alcançada pela agregação de regras com mesmas funções locais no consequente. Ou seja, pela união dos conjuntos aproximados

$$
C R_{7}:\left[\begin{array}{cc}
0,5926 & -0,8055 \\
0,8072 & 0,5902
\end{array}\right] \boldsymbol{x}_{k} \leq\left[\begin{array}{l}
-0,4571 \\
-0,3325
\end{array}\right]
$$

Os cálculos das camadas do modelo TSM são exemplificados a seguir. A variável de particionamento $\boldsymbol{z}_{6,2}$ é calculada por (38), a respectiva funça de perinencia $\mu_{6,2}\left(\boldsymbol{x}_{k}\right)$ por (39), e o

$$
\begin{gathered}
z_{6,2}\left(\boldsymbol{x}_{k}\right)=\left[\begin{array}{ll}
0,6522 & 0,7581
\end{array}\right] \boldsymbol{x}_{k}+0,2203 \\
\mu_{6,2}\left(\boldsymbol{x}_{k}\right)=0,5\left(1-\frac{\left[\begin{array}{ll}
0,6522 & 0,7581
\end{array}\right] \boldsymbol{x}_{k}+0,2203}{\mid\left[\begin{array}{ll}
0,6522 & 0,7581
\end{array}\right] \boldsymbol{x}_{k}+0,2203}\right) \\
w_{6}\left(\boldsymbol{x}_{k}\right)=\beta_{6}\left(\boldsymbol{x}_{k}\right)=\mu_{6,1}\left(\boldsymbol{x}_{k}\right) \mu_{6,2}\left(\boldsymbol{x}_{k}\right) \mu_{6,3}\left(\boldsymbol{x}_{k}\right)= \\
=\mu_{6,1}\left(\boldsymbol{x}_{k}\right) 0,5\left(1-\frac{\left[\begin{array}{ll}
0,65 & 0,76
\end{array}\right] \boldsymbol{x}_{k}+0,22}{\mid\left[\begin{array}{ll}
0,65 & 0,76
\end{array}\right] \boldsymbol{x}_{k}+0,22} \mid\right) \mu_{6,3}\left(\boldsymbol{x}_{k}\right)
\end{gathered}
$$

(1)
e o peso normalizado da regra 6 por (40).

\section{(1)}


$A_{i, j}$ que formam as regiões críticas respectivas a estas regras. No estudo de caso em questão, observe que $\left(A_{6,3}\right)^{\mathrm{c}}=A_{4,1}$ e $\left(A_{6,1}\right)^{\mathrm{c}}=A_{7,1}$ na Figura 3 , além de que $z_{4,1}=z_{6,3}$ e $z_{7,1}=z_{6,1}$. Considerando (26) e as regiões críticas $C R_{4}, C R_{6}$ e $C R_{7}$, define-se a região de saturação positiva $C R_{+}$por (41). Portanto, o peso normalizado desta regra é calculado por (42). O mesmo processo pode ser feito para formar $C R_{\text {- a }}$ partir de $C R_{5}, C R_{8}$ e $C R_{9}$. Assim, tem-se o número de parâmetros do modelo TSM após redução de complexidade na Tabela 1 em [2].

$$
\begin{gathered}
C R_{+}=\left(A_{4,1} \cup A_{4,2}\right) \cap\left(A_{6,1} \cup A_{6,2} \cup A_{6,3}\right) \cap\left(A_{7,1} \cup A_{7,2}\right) \\
=A_{4,2} \cup A_{6,2} \cup A_{7,2}=\left[\left(A_{4,2}\right)^{\mathrm{c}} \cap\left(A_{6,2}\right)^{\mathrm{c}} \cap\left(A_{7,2}\right)^{\mathrm{c}}\right]^{\mathrm{c}} \\
w_{+}\left(\boldsymbol{x}_{k}\right)=1-\left(1-\mu_{4,2}\left(\boldsymbol{x}_{k}\right)\right)\left(1-\mu_{6,2}\left(\boldsymbol{x}_{k}\right)\right)\left(1-\mu_{7,2}\left(\boldsymbol{x}_{k}\right)\right)
\end{gathered}
$$

Tabela 1. Número de parâmetros e operações por método

\begin{tabular}{|c|c|c|c|c|c|c|c|}
\hline \multirow{2}{*}{ Mét. } & \multicolumn{3}{|c|}{ Parâmetros } & \multicolumn{3}{c|}{ Redução \% } & \multirow{2}{*}{$\begin{array}{c}\text { T. proc. } \\
\text { nyyyynn}\end{array}$} \\
\cline { 2 - 7 } & $C R_{i}$ & $f_{i}$ & Tot. & $C R_{i}$ & $f_{i}$ & Tot. & (ms) \\
\hline$[1]$ & 60 & 21 & 81 & 16,7 & 22,2 & 18,2 & 8,6 \\
\hline$[2]$ & 48 & 15 & 63 & 33,3 & 44,4 & 36,4 & 0,2 \\
\hline
\end{tabular}

Note que, na Tabela 1, o modelo TSM [2] requereu menos parâmetros do que a solução explícita [1] após redução de complexidade. Este resultado é explicado pelo fato da notação por conjuntos aproximados ser capaz de representar conjuntos de qualquer formato. Por outro lado, a notação por inequações exige que união das regiões críticas tenha forma de poliedros convexos.

Foram realizadas 200 simulações em malha fechada com as estratégias MPC com solução explícita [1] e com modelo TSM [2]. Os tempos de processamento médios dos métodos durante as simulações são mostrados na Tabela 1. O erro máximo entre os métodos foi da ordem de $10^{-15}$. A evolução dos estados ao longo do tempo é mostrada na Figura 4.

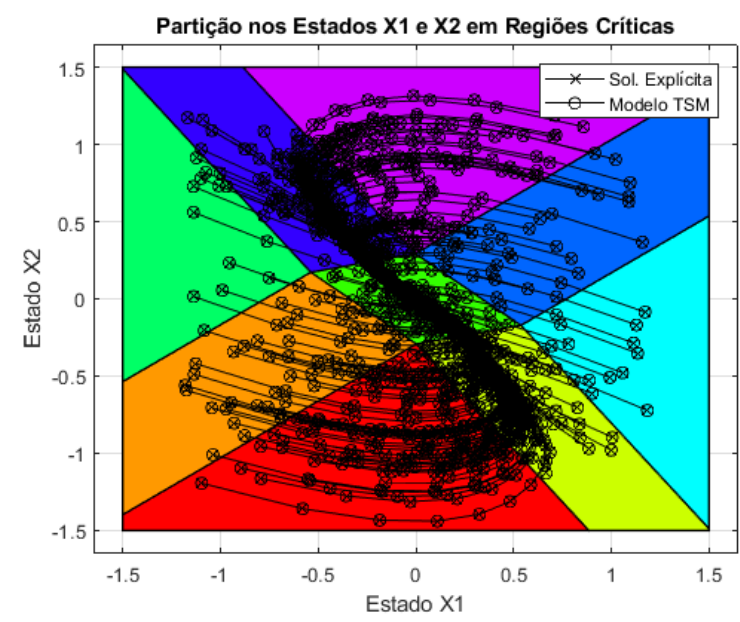

Figura 4- Simulação dos estados para diferentes $\boldsymbol{x}_{0}$, em processador Intel ${ }^{\circledR}$ Core $^{\mathrm{TM}}$ i 7 com $2 \mathrm{GHz}$ de memória RAM.

É possível perceber na Tabela 1 que o modelo TSM [2] alcançou menor tempo de processamento médio do que a solução explícita [1]. Este resultado deve ser analisado a luz da quantidade de operações e do tipo de processamento executado pelos dois métodos. $\mathrm{Na}$ solução explícita [1], o número de operações pode variar, pois o processamento de testes das inequações em (15) é sequencial. O melhor caso acontece quando há sucesso no primeiro teste das inequações de uma região crítica. Enquanto o pior caso ocorre quando há sucesso apenas após o teste de todas regiões criticas, constatando que o estado $\boldsymbol{x}_{k}$ pertence a última região verificada. No modelo TSM, o número de operações não varia e é conhecido a priori. Além disso, exceto por (30), o processamento é realizado em paralelo. Assim, este resultado é explicado pela menor necessidade de parâmetros e pelo processamento paralelo do modelo TSM.

\section{CONCLUSÃO}

Este artigo revisou conceitos de estratégias MPC de solução explícita e modelos TS, além de propor o modelo TSM para calcular esta solução. Este novo método mostrou como vantagens: (i) a capacidade de redução de complexidade das regiões críticas e processamento paralelo, (ii) ausência da fase de treinamento a partir de dados numéricos, e (iii) o mérito de sintetizar uma única expressão analítica para a lei de controle MPC associada. Um estudo de caso comparando foi apresentado. Os resultados mostraram que o calculo pelo modelo TSM necessitou de menor número de parâmetros e tempo de processamento médio do que formulação com solução explícita após redução de complexidade.

Trabalhos futuros serão direcionados para utilização do método proposto em sistemas dinâmicos reais e de diferentes dimensões. Pretende-se usar também o modelo TSM em aplicações com software embarcado e hardware industrial.

\section{AGRADECIMENTOS}

Este trabalho recebeu apoio da CAPES, via acesso do portal de periódicos, e a apoio financeiro da UFRB.

\section{REFERÊNCIAS}

Alessio, A.; Bemporad, A. A survey on explicit model predictive control. Nonlinear model predictive control. Springer, Berlin, Heidelberg, 2009. p. 345-369.

Bemporad, A., Morari, M., Dua, V., e Pistikopoulos, E. N. The explicit linear quadratic regulator for constrained systems. Automatica, v. 38, n. 1, p. 3-20, 2002.

Csekő, L. H; Kvasnica, M. LANTOS, Béla. Explicit MPCbased RBF neural network controller design with discrete-time actual Kalman filter for semiactive suspension. IEEE Transactions on Control Systems Technology, v. 23, n. 5, p. 1736-1753, 2015.

Oberdieck, R., Diangelakis, N. A., Nascu, I., Papathanasiou, M. M., Sun, M., Avraamidou, S., e Pistikopoulos, E. N. On multi-parametric programming and its applications in process systems engineering. Chemical Engineering Research and Design, v. 116, p. 61-82, 2016.

Qin, S.J.; Badgwell, T.A. A survey of industrial model predictive control technology. Control Engineering Practice, v. 11, n. 7, p. 733-764, 2003.

Takagi, T.; Sugeno, M. Fuzzy identification of systems and its applications to modeling and control. IEEE transactions on systems, man, and cybernetics, n. 1, p. 116-132, 1985. 